\title{
Tugendethik und Sozialwissenschaft
}

\author{
Andrew Sayer
}

Online publiziert: 10. August 2018

(C) Der/die Autor(en) 2018

Zusammenfassung In diesem Beitrag vertrete ich die These, dass Tugendethik und Sozialwissenschaft voneinander lernen können. Ich argumentiere, dass diese Zugänge in Bezug auf die Erfassung des Gegenstands der Tugenden komplementäre Stärken und Schwächen haben. Sie lassen sich aber so miteinander verknüpfen, dass ihre jeweiligen Blindstellen ausgeglichen werden können. Ich entwerfe eine solche Verknüpfung im Rekurs auf Bourdieus Konzept des Habitus. Sofern es in einer angemessenen Weise modifiziert wird, erlaubt das Habituskonzept (Un-)Tugenden in ihrem sozialen Kontext zu verorten und deren Ontogenese - insbesondere in der Kindheit - nachzuvollziehen. Obwohl das Habituskonzept als ein soziologisches Konzept entwickelt wurde, trägt es auch zu einem fundierten Verständnis psychologischer Entwicklungsprozesse bei, insbesondere jenen, mit denen sich etwa die Bindungstheorie auseinandersetzt. Darüber hinaus ermöglicht ein Rekurs auf Arbeiten zur Care Ethik die häufig maskulinistischen Engführungen in tugendtheoretischen Ansätzen zu überwinden und zentrale Aspekte menschlicher Vulnerabilität und Care-Bedürftigkeit systematischer zu erschließen. Schließlich argumentiere ich, dass jenes psycho-soziologische Vorstellungsvermögen, das die Sozialwissenschaft bereitstellen kann, ein angemessenes Verständnis von Tugenden und Übeln im Alltagsleben ermöglicht. Dieses Vorstellungsvermögen stellt selbst eine Tugend dar.

Schlüsselwörter Habitus · Tugendethik · Vulnerabilität · Care Ethik · Lebensformen 


\section{Virtue ethics and social science}

Abstract In this contribution, I suggest how virtue ethics and social science might learn from each other. I show that they have complementary strengths and weaknesses regarding virtues, which invite a synthesis that cancels out their respective weaknesses. I outline such a synthesis, centering on Bourdieu's concept of habitus, which, suitably modified, allows us to situate virtues and vices in their social context and understand their development within individuals, particularly in childhood. Although this concept was developed in sociology, it glosses processes of psychological development that are dealt with by attachment theory. Relatedly, the literature on care ethics helps us counter the masculinist bias of most accounts of virtues and to understand human vulnerability and need of care. Further, I argue that having the kind of psycho-sociological imagination that social science provides can help us better to understand virtues and vices in everyday life, and is a virtue in itself.

Keywords Virtue Ethics · Habitus · Vulnerability · Care Ethics · Way of Life

\section{Die komplementären Stärken und Schwächen der Philosophie und Sozialwissenschaften}

Philosophie und Sozialwissenschaften haben sich in den letzten 150 Jahren voneinander entfremdet. Beide Disziplinen sind dadurch erheblich in Mitleidenschaft gezogen worden. Denn die Trennung normativen Denkens von der empirischen Sozialwissenschaft hat sowohl das Verständnis der Philosophie darüber reduziert, wie Praktiken mit gesellschaftlichen Zusammenhängen und Verortungen verbunden sind, als auch das sozialwissenschaftliche Verständnis der normativen Qualität leibhaftiger sozialer Erfahrungen. Daher trägt die Sozialwissenschaft derzeit wenig zu Fragen der ethischen Dimensionen von Lebensformen bei. Sie tut sich schwer damit, zu erklären, warum Menschen überhaupt irgendetwas wichtig ist (Sayer 2011). In der sozialwissenschaftlichen Methodologie finden sich Versuche, Wertfragen zu verdrängen sowie - dies wird weniger beachtet - Vernunft und Wertfragen zu trennen, während seitens der Philosophie substanzielle sozialwissenschaftliche Einsichten unberücksichtigt bleiben. Wo Philosph*innen dazu tendieren, Menschen als selbstbestimmte, rationale Individuen zu konzipieren, die Dinge tun wie z.B. ein „Lebensprojekt“ zu wählen, tendieren Anthropolog*innen und Soziolog*innen oft dazu, Individuen Entscheidungsfreiheiten abzusprechen ${ }^{1}$. Die Fragmentierung der Sozialwissenschaft in unterschiedliche Disziplinen hat diese Situation verschlimmert. Ökonomie, Soziologie, Psychologie etc. nehmen sich wechselseitig misstrauisch und z. T. auch mit Geringschätzung zur Kenntnis, obwohl sie de facto aufeinander angewiesen sind.

\footnotetext{
${ }^{1}$ Dabei formulieren sie ein Subjektverständnis, das sie bemerkenswerterweise auf sich selbst nicht anwenden.
} 
Die Attraktivität der Tugendethik für die Sozialwissenschaft besteht nun darin, dass sie, anders als z. B. der Utilitarismus, deontologische oder sozial-kontraktualistische Ansätze, zumindest ein gewisses Verständnis von gesellschaftlichen Einflüssen hat, die auf uns als ethische Wesen wirken und dass sie - über Fragen der Vernunft hinaus - die Relevanz von Erfahrungen, Praktiken und Verinnerlichung anerkennen. Sie beschäftigt sich mit leibhaftigen, menschlichen Wesen und mithin mit Wesen, die durch ihr Aufwachsen und durch ihre Umstände geformt sind.

$\mathrm{Ob}$ als Forschungsgegenstände oder in Bezug auf ihre Methodologie: Sozialwissenschaften hadern mit Wertfragen. Gesellschaftlich vorfindbare Werte werden tendenziell entweder als ,lediglich subjektiv“ betrachtet (als ob sie auf nichts bezogen und nicht in der Lage wären, mehr oder weniger zutreffend zu sein) oder als Effekt von Konventionen, als willkürliche Regeln ohne besonderen moralischen oder wohlergehensförderlichen Inhalt. Sie werden häufig als ir- oder a-rational betrachtet und folglich als etwas, dass dem Anspruch auf Objektivität oder rationale Argumentation entgegensteht. Sowohl Konservative, die das vermeintliche „Eindringen“ von Werten vermeiden wollen, um Objektivität sicher zu stellen, als auch Radikale, die argumentieren, nicht wertfrei zu sein und deshalb erst gar keinen Objektivitätsanspruch formulieren, teilen die selbe Fehlannahme: Sie gehen davon aus, dass sich Werte und Objektivität ausschließen.

Es gibt zahlreiche, miteinander verbundene Gründe für die Vermeidung von Wertfragen:

a) Eine verbreitete und sehr ernsthafte Befürchtung ethnozentristisch zu sein.

b) Eine Ablehnung naturalistischer Erklärungen von Gut und Böse, die mit einer Art bio-phobischem Kulturalismus oder einem überbordenden Soziologismus verknüpft ist.

c) Eine Unwilligkeit, ,,wertend“ zu sein, wenngleich die Kritikwürdigkeit bestimmter Einstellungen - wie etwa Rassismus, Sexismus und Homophobie - kaum bestritten wird.

d) Eine Tendenz zur Annahme, dass Normativität lediglich aus präskriptiven Handlungsaufforderungen bestehe. Dies mündet in der Annahme, dass mit der SeinsSollens-Unterscheidung alles gesagt sei, was es über Wertfragen zu wissen gelte. Dabei wird die zentrale Rolle von evaluativen Beschreibungen und Bewertungen im Kontext von Normativität ignoriert.

e) Eine negative Wahrnehmung von Moral als etwas Repressivem.

Was im sozialwissenschaftlichen Diskurs als Normativität betrachtet wird, ist jedoch in einem hohen Maße selektiv. Die zeitgenössische britische Soziologie betont z.B. Übel, die mit Diskriminierungen auf der Basis askriptiver Gruppenmerkmale verbunden sind (Rassismus, Sexismus, Homophobie etc.) sowie Gewalt, Unterdrückung und Ausbeutung. Typischerweise - und seitenverkehrt zur üblichen Perspektive in der Philosophie - werden jedoch Tugenden lediglich implizit thematisiert und in der Regel als die bloße Abwesenheit solcher Übel verstanden. Doch ohne Zweifel kann auch jemand, der nicht rassistisch, nicht sexistisch etc. ist, dennoch selbstsüchtig oder hartherzig sein, vorausgesetzt, diese Haltungen sind nicht spezifisch auf Race, Gender, Sexualität etc. bezogen. Es ist selten, dass die Soziologie Tugenden wie Großzügigkeit, Mitgefühl, Freundlichkeit oder Liebe in den Blick 
nimmt. Vielmehr werden Bezüge auf „Tugenden“ skeptisch betrachtet und gelten als unangemessene „Moralisierung“. Dieser Ansicht korrespondiert eine Präferenz für eine unbeteiligte, distanzierte Perspektive auf Individuen, deren Deutungen und Gefühlen oft wenig Beachtung geschenkt wird. Stattdessen werden sie als Produkte vorherrschender Diskurse, Machtkonstellationen und Umstände verstanden. Selbst noch Sozialwissenschaftler*innen, die auf Konzepte der „Affektualität“ rekurrieren, finden anti-humanistische Wege, dies zu tun und Individuen als ,affect dopes“ zu behandeln. Wie ich an anderer Stelle ausgeführt habe, tragen solche Tendenzen zum entfremdeten und entfremdenden Charakter eines großen Teils der gegenwärtigen Sozialwissenschaft bei (Sayer 2011, 2005).

Nun ist es zu begrüßen, dass sich die Sozialwissenschaft mit Übeln wie z.B. Sexismus auseinandersetzt, aber sie versäumt es, sich darüber hinaus auch mit Tugenden zu beschäftigen. Seitenverkehrt irrt die Philosophie, wenn sie Übel als weniger wichtig oder als bloße Abwesenheit des Guten betrachtet. Übel sind aktiv und keine bloße Absenz (Vetlesen 2005; Glover 2001). In der lebensweltlichen Wirklichkeit sind Tugenden und Laster oft eng verknüpft. Wenn die Philosophie Rassismus, Sexismus, Unterwerfung, Ausbeutung usw. ignoriert und uns auf Tapferkeit, Dankbarkeit, Mitgefühl usw. reduziert, macht sie sich individualistisch, voluntaristisch, affektiert, apolitisch und belanglos - allerdings gerade darum politisch umso nützlicher. In ihrer gegenwärtig fragmentierten, beschränkten und entfremdeten Form weisen Philosophie und Sozialwissenschaften insofern komplementäre Stärken und Schwächen auf.

\section{Versuch einer Synthese}

Um diese Probleme zu überwinden, schlage ich vor, was man als qualifizierte, ethisch naturalistische Perspektive auf Normativität beschreiben kann. Diese Perspektive behauptet nicht, Natur sei an sich gut oder schlecht, wohl aber, dass Entfaltung (Flourishing) und Leiden (Suffering) sowie die Bedeutung des Guten und Schlechten in letzter Instanz mit der Conditio Humana verbunden sind. Diese Behauptung lässt sich weiter qualifizieren:

- Flourishing und Suffering sind objektive Zustände, die (in fallibeler Weise) erfasst werden können. Flourishing ist dabei nicht nur die Abwesenheit von Leiden, sondern mit der Verfolgung unserer (Selbst-)Verpflichtungen und die Entwicklung unserer Fähigkeiten verbunden.

- Kultur beeinflusst zwar körperliche und mentale Zustände, dies geschieht aber innerhalb natürlicher Grenzen: Wir verfügen unterschlich akkulturierte Naturen, die vielfältige Formen von Flourishing und Suffering annehmen können. Was ich daher vorschlage, ist ein objektivistisches aber pluralistisches Verständnis von Wohlergehen.

- Bedürfnisse, Flourishing und Suffering sind immer kulturell interpretiert. Entsprechend finden sich vielfältige und unterschiedliche Konzeptionen von Bedürfnissen, Flourishing und Suffering. Aber diese Vorstellungen und Interpretationen sind fallibel. 
- Einige Güter sind substanziell kulturell konstruiert - etwa die internen Güter von „Praktiken“ im Sinne MacIntyres (1981) - und fungieren als eigenständige emergente Quellen von Flourishing (vgl. MacIntyre 1981).

Mit dieser Perspektive sind bestimmte ontologische Annahmen über Menschen verknüpft. Kurz:

- Wir sind rationale Wesen, die sowohl psychologisch als auch sozial von anderen abhängig sind.

- Wir sind Beziehungswesen: Unser Selbstbild, unsere Vernunftfähigkeit und unsere Fähigkeit zu psychischem Wohlergehen hängt von unseren Beziehungen zu anderen $\mathrm{ab}$.

- Wir sind sowohl verletzlich als auch handlungsfähig. Unsere gegenwärtigen Zustände von Flourishing und Leiden werden stets in verändere Zustände überführt. Wir bewegen uns permanent zwischen den Dingen wie sie sind und wie sie sein könnten.

- Wir leben am Rande des Mangels. Wir sind fähig aufzusteigen und zwar häufig, in dem wir neue Formen entwickeln, um uns zu schützen und uns zu entfalten. Um ein Abrutschen zu verhindern, sind wir notorisch auf Versuche verwiesen, unsere Situationen zu verteidigen und zu verbessern.

- Vor dem Hintergrund unserer Bedürftigkeit und Verwundbarkeit sowie unserer Möglichkeit, uns zu entfalten, ist unsere Beziehung zur Welt eine Beziehung von „Concern“. Dabei agieren wir als evaluative Wesen.

- Unsere Care-Bedürftigkeit ist universell.

- Physiologische und psychologische Heterogenität ist normal.

- Unsere körperlichen, intellektuellen und geistigen Entwicklungen sind stets eine Folge der Interaktionen mit unserer sozialen und materiellen Umwelt. Diese Interaktionen werden durch unsere je unterschiedlichen Kräfte (Powers) und Schwächen ermöglicht und beschränkt.

\section{Habitus, Praxis und Tugendenden}

Das Werk von Pierre Bourdieu schlägt eine fruchtbare Brücke zwischen sozialwissenschaftlichen Ansätzen zur Erklärung von Verhalten und der Tugendethik. Dies gilt, obwohl sich Bourdieu selbst nur wenig für Ethik interessiert hat.

Von allen Disziplinen ist die Philosophie am anfälligsten für das, was Bourdieu (2000) als ,,scholastischen Fehlschluss“ bei der Interpretation sozialer Praktiken bezeichnet. Dieser Fehlschluss besteht in einer akademischen Projektion der eigenen kontemplativen, diskursiven und analytischen Beziehungen zur Welt auf andere, deren primär praktische Weltbeziehungen übersehen oder entwertet werden. Der scholastische Fehlschluss tendiert dazu, Handlungen auf ein Resultat von Vernunfterwägungen oder Diskursen zu reduzieren und verinnerlichte Dispositionen, Gefühle, Neigungen, Fähigkeiten und das „Gefühl für das Spiel“ zu ignorieren sowie dazu, geistige Aktivitäten auf Deliberation zu verkürzen. Bourdieus Überlegungen können insofern ergänzt werden, dass der scholastische Fehlschluss auch dazu tendiert, 
Gefühle als das Gegenteil von Vernunft misszuverstehen und Köper als heteronome Gegenstände oder bloße Träger von und gegebenenfalls auch als Hemmnis für geistige Aktivitäten zu verstehen. Er beinhaltet ferner eine kühle, distanzierte und szientistisch verkürzte Auseinandersetzung mit Vulnerabilität, Abhängigkeit, Gefühlen, Liebe und Care.

Ein Aspekt, der die Tugendethik für die Sozialwissenschaft attraktiv macht, ist deren Betonung der Bedeutung von Dispositionen und Erfahrungen. Dies macht sie für scholastische Fehlschlüsse weniger anfällig als andere Ansätze der Moralphilosophie. Dennoch bleibt auch sie anfällig für Idealisierungen, die das Resultat eines dekontextualisierten Verständnisses von Tugenden sind, das wesentliche soziale Strukturen ignoriert, die Individuen und die Wirkungen ihrer Praktiken beeinflussen.

Bourdieus bekanntestes Konzept ist das des „Habitus“ - ein Konzept, dass Affinitäten zu Aristoteles aufweist. Der Habitus ist ein eingeschriebenes Bündel von Dispositionen, die Individuen durch wiederholte Praxis und Erfahrung (insbesondere in frühen Lebensphasen - den „formativen Jahren“) entsprechend ihrer Lebenswelt bzw. ihrer Stellung im sozialen Feld erwerben. Diese Dispositionen eines Individuums sind an dessen spezifische Erfahrungen, Umwelten und sozialen Beziehungen angepasst. Individuen entwickeln so ihr „Gefühl für das Spiel“, das ihnen in ungewohnten Situationen fehlt. Kinder aus dem Bildungsbürgertum werden etwa daran gewöhnt, Diskussionen über das Zeitgeschehen zu hören und sich daran zu beteiligen. Dabei lernen sie den Standpunkt von Akteur*innen, die über andere verfügen können, die erwarten können, dass ihnen zugehört wird und mit denen es sich mithin lohnt, über politische Sachverhalte zu diskutieren. Entsprechend werden sie häufig redegewandt, meistern Übergänge in Schulen und Universitäten vergleichsweise problemfrei und entwickeln typischerweise einen selbstverständlichen „Berechtigungssinn“ (sense of entitlement and ease), der ein Effekt ihrer sicheren ökonomischen Position und ihres Status ist. Demgegenüber ist es für Kinder in einkommensschwachen Familien wahrscheinlicher, Dispositionen zu erwerben, die an den Umstand angepasst sind, über wenig Macht und Status zu verfügen und mit schwierigen Umwelten zurecht zu kommen. Für sie ist es wahrscheinlicher als für Mittelschichtskinder, gewieft und zäh aber weniger sprachgewandt zu sein. Sie haben tendenziell niedrigere Erwartungen, was auch den Umstand reflektiert, dass die Jobs, die sie wahrscheinlich ausüben werden, eher im Erfüllen von Anweisungen bestehen und eher wenig Ermessens- und Entscheidungsspielraum beinhalten ${ }^{2}$. Der Habitus hat nicht nur eine klassenspezifische, sondern auch eine gegenderte Form als Resultat der Involvierung in genderspezifische Praktiken. Dabei strukturiert die Struktur des sozialen Feldes, in dem Menschen sich - insbesondere in ihren frühen Lebensphasen - überwiegend bewegen, deren Dispositionen und Sichtweisen. Zumal ihre Praktiken mit diesen Dispositionen verknüpft sind, tendieren sie dazu, dieses Feld von Praktiken zu reproduzieren. Dennoch kann sich der Habitus eines Individuums verändern. Die Veränderung der Dispositionen, die den Habitus konstituieren, setzt jedoch Zeit und wiederholte Praktiken voraus. Auch Aufwärtsmobilität ist möglich, allerdings fühlen sich oft auch jene, deren Erfolg und Kompetenzen evi-

\footnotetext{
2 Auch wenn es sich dabei um Verallgemeinerungen handelt, von denen es zahlreiche Ausnahmen gibt, ist dies der Befund sozialwissenschaftlicher Studien (Lareau 2003; Reay 2002, 1998).
} 
dent sind, fehl am Platze und nicht zugehörig. Dies ist ein Effekt der Historizität des Habitus.

Bourdieu betrachtete alltägliche Praktiken als überwiegend halb-bewusst, routiniert und mit einem ausgebildeten Gefühl für das Spiel verknüpft. Eine Reihe von Kritiker*innen hat moniert, dass im Habituskonzept ein zu deterministisches Praxisverständnis angelegt sei. Diese Kritik tendiert jedoch dazu, die generative und kreative Kraft verkörperlichter Dispositionen zu übersehen. Diese sind lernfähig, in der Lage zu improvisieren und kreativ mit einem gewissen Maß an Variationen umzugehen. Sie lassen sich als etwas verstehen was Expert*innen von Anfänger*innen unterscheidet. Es gibt keine zwei Tennisspiele und eben auch keine zwei soziale Situationen, die identisch sind. Dieser Aspekt ist auch in philosophischen Werken zur praktischen Vernunft (Dunne 1993) und in Studien zur Aneignung praktischer Fähigkeiten (Benner 1994) herausgearbeitet worden. Die Fähigkeiten versierter Musiker*innen, Fußballspieler*innen oder sozial kompetenter Schauspieler*innen entspringen aus ihrem praktischen Sinn und ihren erworbenen Dispositionen und nicht einfach aus einem überlegenen Zugang zu ihren jeweiligen Kompetenzen. Ohne einen solchen praktischen Sinn wären wir geradezu hilflos und zu viel Selbst-Besinnung und Selbstanalyse kann unsere Fähigkeit zu erfolgreichem Handeln einschränken. Nicht von ungefähr kommen wir uns „,blöd“, überfordert und handlungsunfähig vor in sozialen Situationen, in denen uns der praktische Sinn für das Spiel fehlt.

Es mag einer Überkorrektur des scholastischen Fehlschlusses geschuldet ein, der Bourdieu dazu verleitet, das Ausmaß zu unterschätzen, in dem der Erwerb dieser Dispositionen reflexiv durch die Individuen vermittelt und durch Diskurse und Bildung - inklusive moralischer Geschichten und Imaginationen - beeinflusst wird. Menschen passen sich nicht einfach in einer osmotischen Weise an bekannte Situationen an. Vielmehr nehmen sie prüfende und moralische Bewertungen vor. Sie betrachten bestimmte Verhaltensweisen und Situationen als fair oder unfair, anständig oder unanständig usw. Die diskursiven und materiellen Einflüsse mit denen sie konfrontiert sind, stellen sich in der Regel als unterschiedliche Optionen oder konfligierende Zwänge dar, auf die es typischerweise alternative Antwortmöglichkeiten gibt. Entsprechend leidet oder gedeiht auch das „habituierte“ Individuum auf eine vielfältige Weise und es ist sich dessen auch in unterschiedlichem Maße bewusst. Unsere fortwährende Wertung und Gewichtung erfolgt häufig halb-bewusst. Mit Iris Murdoch (1970) kann man sie als Teil der „Aufmerksamkeitsarbeit“"verstehen, die auf ,structures of value around us“ aufbaut, bei der ,,at crucial moments of choice most of the business of choosing is already over" (Murdoch 1970, S. 36). Die Gewöhnung an das Verhalten anderer und Muster bekannter Praktiken neutralisiert aber nicht zwangsläufig evaluative Beurteilungen und Gefühle. Die wiederkehrende Erfahrung von respekt- und rücksichtslosem Verhalten kann dauerhaft verärgern, sie kann zu Verbitterung, geringem Selbstwert, Belastung und Scham aber auch zu aktiven Widerstand führen - obwohl Widerstand, wie uns Bourdieu erinnert, schmerzhaft und kostspielig ist (dazu auch Tessman 2005).

Wie Tugendethiker*innen darüber hinaus betonen würden, handelt das tugendhafte Individuum zwar spontan - z. B. indem es einer alten Person, die gestürzt ist unmittelbar zu Hilfe kommt - aber es ist sich auch bewusst, dass dies zu tun ,das Richtige“ ist und ist in der Lage, dies zu rechtfertigen. Insofern gibt es, obwohl 
Bourdieu selbst dies nicht ausführt, ein eudaimonistisches Moment im Habitus. Der Habitus ist kein normativ indifferentes „Ding“.

Die verinnerlichten Dispositionen, die unseren erworbenen Habitus ausmachen, spiegeln unsere spezifischen Positionen im sozialen Feld in Bezug auf andere Individuen, Gruppen und Institutionen. In der Philosophie wird die Tatsache, dass dieses Feld in modernen Gesellschaften durch Ungleichheit geprägt ist, oft ignoriert. Es findet sich eine hierarchische Streuung von Positionen der Dominanz und Subdominanz sowie damit korrespondierende Dispositionen der Herablassung und Ehrerbietung, Überlegenheit und Unterlegenheit, Berechtigung und „Wissen-wo-man-hingehört“, Stolz und Scham, Gelassenheit und Unsicherheit usw., die auf weite Teile unserer Erfahrungen abfärben. Diese Dispositionen und Ungleichheiten finden in den meisten philosophischen Debatten auffallend wenig Beachtung: Wenn Gesellschaften als Aggregate von Individuen missverstanden werden, die nur von ihren eigenen Entscheidungen und „Glück“ (luck) beeinflusst werden, geraten klassen-, gender- und race-spezifische strukturelle Ungleichheiten aus dem Blick.

Obwohl Bourdieu ein sehr aufmerksamer Beobachter habitueller Dispositionen war, hat er deren emotionale und ethische Dimensionen weitgehend ignoriert. Entsprechend hat er das, was er als die Konkurrenzen und Kämpfe im sozialen Feld beschreibt, eher in einem Hobbesianischen, instrumentell orientierten Sinne verstanden und nicht (auch) als Kämpfe um die Art, wie man leben soll (Bourdieu 1986). Das soziale, kulturelle und ökonomische Kapital der dominanten Klasse sorgt für kompetitive Vorteile. Aber es geht nicht nur um Kämpfe um solche Vorteile, sondern auch um Kämpfe darüber, was - welche Güter, Verhaltensweisen, Lebensführungen oder Praktiken - als wertvoll gilt und was als wertvoll gelten sollte. Anders formuliert geht es bei den Kämpfen im sozialen Feld nicht nur um instrumentelle, kompetitive Vorteile, sondern auch um den intrinsischen Wert von Gütern.

Davon unabhängig sind die Bewertungen anderer und deren Handlungen tendenziell von den relativen Positionen im sozialen Feld abhängig. Sie sind in der Regel mit gender- und klassenspezifischen Annahmen und jenen Beziehungen der Herablassung und Fügsamkeit, des Ressentiments, Neides und der Schuld assoziiert, die mit Klassenungleichheiten verbunden sind. Wie Adam Smith (1759/1984) in einer viel zitierten Bemerkung ausgeführt hat, können moralische Gefühle gegenüber anderen durch die Ungleichheiten des sozialen Feldes deformiert werden: „This disposition to admire, and almost to worship, the rich and the powerful, and to despise, or at least to neglect persons of poor and mean condition [...] [is] the great and most universal cause of the corruption of our moral sentiments" (Smith 1984 [1759], S. 61; dazu auch Tessman 2005).

Während, wie Bourdieu in seinen „feinen Unterschieden“ nachzeichnet, der ästhetische Geschmack von Individuen im Großen und Ganzen mit ihren Positionen im sozialen Feld korrespondiert, können wir, im Anschluss an Smith, davon ausgehen, dass dies für moralische Gefühle weniger gilt. Dies ist gerade deshalb der Fall, weil sie unsere Beziehungen zu Anderen betreffen, seien sie nun Teil unserer eigenen Gruppe oder nicht. Moralische Gefühle haben eine inhärent generalisierende Tendenz - was gut oder richtig ist, gilt nicht nur für uns, sondern auch für andere. Es gibt eine Reihe von Dispositionen, die deutlich mit unserer sozialen Position zusammenhangen, wie etwa, die sich zu fügen und zu dienen, oder zu bestimmen 
und bedient zu werden, oder zu reflektieren statt ,lediglich irgendwie klar zu kommen“. Es gibt aber auch Dispositionen und Empfindungen wie etwa Wohlwollen oder Missbilligung von Ungerechtigkeit, die über einzelne soziale Felder hinweg verbreitet sind, selbst wenn die Kontexte unter denen sie hervorgebracht werden individuell differieren. Ungeachtet der verbreiteten „Korrumpierung“ unserer moralischen Gefühle durch Ungleichheitsbeziehungen, oder wie es Lisa Tessman (2005) formuliert, die „Belastung von Tugenden“, ist es für die Voreingenommenen, die mit Verhalten korrespondiert werden, das ihrem Vorurteil nicht entspricht, möglich, solche Ausnahmen wahrzunehmen.

Unsere Position im sozialen Feld beeinflusst was wir sind. Dies kann als konstitutives moralisches Glück beschrieben werden (Griswold 1999, S. 241). Wie es Martha Nussbaum formuliert: ,much that I did not make goes towards making me whatever I shall be praised or blamed for being " (Nussbaum 1986, S. 5). Dies deckt sich mit einer Formulierung aus der Bibel: „Was hast du, das du nicht empfangen hast? Wenn du es aber empfangen hast, was rühmst du dich dann, als hättest du es nicht empfangen?" (1 Korinther 4, 7). Gleichwohl bleiben wir in einem bestimmten Ausmaß verantwortlich für unsere Persönlichkeit: Wir sind, wie es Aristoteles formuliert, „teil-ursächlich“. Aber die individualisierenden Tendenzen in Alltagserklärungen von Verhalten und Beurteilungen von Personen in westlichen Kulturen die durch die Betonung von Freiheit und Autonomie verstärkt werden - tragen dazu bei, dass sowohl das konstitutive moralische Glück als auch die generative Relevanz sozialer Kontexte unterschätzt werden. Dies trägt zu irrigen moralischen Bewertungen Anderer bei. Die privilegierte Person, die sich vorstellt, wie sie für ihren Aufstieg kämpfen würde, wenn sie in eine arme Familie geboren wäre, verkennt, dass sie eine andere Person wäre, wenn sie einen solchen Start ins Leben gehabt hätte. Dabei sollten wir uns auch vor romantischen Mythen hüten, die sich teilweise auf Seiten der Linken finden: Die ,Salz-der Erde-Romantik“, dass Armut und geteiltes Elend Solidarität und Güte hervorbringen. Das mag ja im Einzelnen der Fall sein, aber wie Martha Nussbaum (2001) kommentiert: „,Wenn wir verstehen, dass sich die Erfahrung von Ungerechtigkeit in die Persönlichkeit der Betroffenen selbst einschreiben und Wut, Missgunst und das Fundament schlechter Charaktereigenschaften hervorbringen kann, sollten wir umso mehr bemüht sein, jedem Kind die materielle und soziale Unterstützungen zuteilwerden zu lassen, die die Voraussetzungen für menschliche Würde sind“ (Nussbaum 2001, S. 414). Armut und Stigma veredeln Menschen nicht. Sie und nicht die daraus erwachsenden moralischen Problematiken sind es, die zuvörderst korrigiert werden müssen: Den Armen mangelt es an Einkommen und Arbeit, nicht an „Resilienz“ oder „Persönlichkeit“. Wir brauchen daher eine sozialwissenschaftliche Vorstellung, die geeignet ist, Tugend und Übel gesellschaftlich zu verstehen. Tatsächlich ist ein solches Empfindungsvermögen selbst eine Tugend im Alltagsleben. Ein Verständnis von Tugend und Persönlichkeit ist zwar wichtig, es ist aber notwendig dieses Verständnis an der richtigen Stelle zu platzieren.

Wir brauchen verstärkte Forschung darüber, wie real-existierende Formen sozialer Organisation Tugenden und Übel verstärken oder abschwächen. Leute tendieren dazu, weniger Empathie für Menschen am unteren Rand aufzubringen je stärker die gesellschaftliche Ungleichheit ausgeprägt ist. Wo bestimmte Gesellschaften unglei- 
cher werden, zeigen soziale Einstellungen eine abnehmende Sorge um Ungleichheit. Zusätzlich zu Ungleichheiten tragen bestimmte Organisationsformen zur Genese bestimmter Tugenden oder Übel bei. Es ist viel darüber geschrieben worden, wie neoliberale Ökonomien Egoismus und mangelndes Interesse am Gemeinwohl befördern und wie Audits und leistungsbezogene Besoldung professionelle Ethiken schwächen (Brennan und Pettit 2004; Pink 2011).

Selbstverständlich können Leute Ungleichheiten auf unterschiedliche Weisen deuten, abhängig von ihren jeweiligen Werten und Interpretationen, aber Ungleichheiten selbst tendieren - wie Smith beobachtet hat - dazu, moralische Urteile sich selbst und anderen gegenüber zu deformieren.

\section{Psycho-soziale Prozesse und Care}

Obwohl das Habituskonzept ein soziologisches ist, setzt es neurologische und psychologische Prozesse voraus, die die Formation von Dispositionen ermöglichen. Das Habituskonzept ist ein Platzhalter für Habitualisierungsprozesse und die Entwicklung praktischen Wissens. Diese setzten u. a. jene Neuroplastizität voraus, die traditioneller Gegenstand psychologischer Forschungen ist. Statt eifersüchtig ihre Königreiche zu verteidigen, sollten diese Disziplinen daher zusammenarbeiten. Als eine naturalistische Philosophie muss auch die Tugendethik die Frage, wie sich Tugenden und Übel entwickeln, als eine empirische Frage berücksichtigen und sich entsprechend auch gegenüber Befunden neurologischer und psychologischer Forschungen öffnen.

Die Bindungsforschung erweist sich diesbezüglich als besonders relevant. Frühe Bindungen zu primären Care-Givern sind von zentraler Bedeutung für unsere spätere Entwicklung und unser Wohlergehen. Das Streben des Kindes nach Bindung ,hilft dem unreifen Gehirn die reifen Funktionen des Gehirns der Eltern zu nutzen um seine eigenen Prozesse zu organisieren“ (Siegel 1999, S. 67). Wie das Gehirn eines Säuglings organisiert wird, hängt insofern von der Art der Interaktion mit primären Care-Givern ab: Vernachlässigung oder Über-Protektion, Ängste oder Gewalt können dauerhafte und schwer zu verändernde Problematiken evozieren, die die sozialen Fähigkeiten des Kindes im späteren Leben beeinträchtigen (Cassidy und Shriver 1999; Benjamin 1988; Bowlby 1969; Winnicott 1964) inklusive der Fähigkeiten zur Funktion als eigenständig denkendes Wesen. Von zentraler Bedeutung ist dabei die vorsprachliche Phase: Unser Selbstverständnis hängt von unseren Erfahrungen ab, gehalten zu werden, geliebt zu werden, dass mit uns gespielt wird, dass wir begehrt statt ignoriert, abgelehnt, verletzt beschämt usw. werden. Der Bindungsprozess umfasst den ganzen Körper und nicht nur kognitive Dimensionen. Der Fokus der Philosophie auf Diskurse und Vernunft droht, diese wichtigen Einflüsse auf die Art der Person zu übersehen, zu der wir uns entwickeln. Sich mit Säuglingen und diesen grundlegenden Bedürfnissen auseinanderzusetzen mag in einem Spannungsverhältnis zu dem maskulinen akademischen Habitus und zur professionellen Gravitas stehen, aber wir müssen dies ernst nehmen, wenn wir uns für ,human flourishing" interessieren. 
Ob wir sie nun als Teil der Tugendethik verstehen oder nicht, sind schließlich auch die Arbeiten zur Care-Ethik von höchster Relevanz, um Tugenden und die moralische Qualität des Alltagslebens zu verstehen (Lynch et al. 2009; Held 2006; Kittay 1999; Sevenhuijsen 1998; Tronto 1994). Unser Bedarf an Care ist universell: Ohne Care gäbe es uns nicht. Care sollte daher im Zentrum und nicht am Rande von Ethiken stehen. Als relational abhängige Wesen ist unsere Abhängigkeit von Anderen physiologischer, psychologischer und sozialer Natur und Abhängigkeit kann gedeihliche wie schädliche Formen annehmen: Wir sollten uns daher der Tendenz entgegenstellen, Vulnerabilität und Abhängigkeit von anderen als einen Mangel statt als Teil der Conditio Humana zu verstehen, den wir anerkennen müssen, um mit uns selbst und anderen zu harmonieren. Dies erfordert, die maskulinistischen Scheuklappen abzunehmen, die sich in üblichen Tugendlisten und in der liberalen Betonung von Autonomie finden und stattdessen Care wertzuschätzen, insbesondere die Arbeit des Sorgens für (im Gegensatz zum Besorgt-Sein über) Andere. Die Disposition zu und Praxis von Care für Andere ist selbst eine Tugend.

Eine zentrales Argument der Care-Ethik besteht nun darin, dass Care nicht nur einzelne oder auch wiederholte Akte des Wohlwollens und der Unterstützung, sondern die Pflege einer besonderen Beziehung der sorgenden und der Person umfasst, auf die sich die Sorge bezieht. Damit erkennt die Care-Theorie an, was liberale Moraltheorien häufig ignorieren, nämlich den konstitutiven Charakter von Beziehungen zu signifikanten Anderen für das, was Individuen sind und derer unerlässlich für ihr Selbstverständnis. Dabei lässt sich die Pflege von Selbst und Anderen weder auf Eigennutz noch auf Altruismus reduzieren: Die Aufrechterhaltung und Entwicklung solcher Beziehungen beinhaltet nicht immer Selbstaufopferung (Held 2006), sondern ist wesentlich für flourishing.

Allerdings steht die Anerkennung solcher Formen von Care in einem Spannungsverhältnis zu der Priorisierung jener Unparteilichkeit, auf der Gerechtigkeitstheorien in der Regel aufbauen. Da es nun aber kaum als ethisch gelten kann, z. B. Kindern jene unkonditionale Liebe und dauerhaften Bindungen vorzuenthalten, die zentraler Bestandteil von Wohlergehen sind, ist es notwendig, diese beiden Aspekte in irgendeiner Weise theoretisch und praktisch zu versöhnen. Eine Möglichkeit hat Richard Norman vorgeschlagen: „It is because we have specific commitments to specific individuals and groups that we can then go on to recognize the claim of all human beings [...]. It is because we first form ties with parents, siblings and friends that we are subsequently able to extend our sympathies to other human beings with whom we are less closely connected" (zit. nach Goodin 1985, S. 4).

Um die Bedeutung Anderer anzuerkennen bedarf es nicht nur eines intellektuellen Verantwortungsbewusstseins sondern auch der Empathie. Die Anerkennung des normativen Werts der Pflege von Beziehungen und des Mit-Anderen-Seins durch die Care-Ethik markiert eine radikal andere Perspektive als die liberale Perspektive auf den erwachsenen Mann, der das Subjekt von Gerechtigkeitsforderungen in so vielen Gerechtigkeitstheorien ist - das Individuum, das virtuos in der Auseinandersetzung mit spezifischen Situationen handelt, aber in respektvoller Distanz zu Anderen bleibt und für das die konstitutiven und gegebenenfalls intimen Liebesund Care-Beziehungen sowie enge Freundschaften nebensächlich oder privat und mithin jenseits theoretischer Relevanz bleiben. Die Care-Ethik steht daher in einem 
Spannungsverhältnis sowohl zu dem Vorrang, den die Philosophie der Vernunft, der freischwebenden Reflexion und dem Zweifel einräumt als auch zu der akademischen Wertschätzung individueller Leistungen.

Eine weitere Stärke der Arbeiten zur Care-Ethik besteht darin, dass sie CareArbeit in Machtfeldern situieren, die jene gender-typischen Formen hervorbringen, die es wahrscheinlicher machen, dass weibliche Care-Arbeiterinnen durch überproportionale Care-Verpflichtungen entmachtet werden und Erfahrungen von Gefühlen der Frustration, des Zorns und der Schuld machen. Während die Idealisierung der Care-Arbeitenden als ,ein Engel“ eine patriarchale Form darstellt, dies auszublenden, trägt Philosophie zu einer Entpolitisierung dieser Problematik bei, wenn sie von solchen Kontexten abstrahiert.

Die Tugendethik sollte daher die Einsichten der Care-Ethik und der Sozial-Psychologie der Bindungen aufnehmen. Sie muss über die normative Reflexion über die Beschaffenheit von Tugenden ebenso hinausgehen wie über eine lediglich symbolische Anerkennung der Wichtigkeit von Bildung für deren Erwerb.

Die Rede von Tugenden und Lastern impliziert häufig die Annahme einer konsistenten Praxisweise. Empirisch legen psychologische Untersuchungen aber nahe, dass ethische und unethische Verhaltensweisen durch situative Stimmungen beeinflusst werden: Menschen sind eher bereit, anderen zu helfen, wenn sie gerade erfreuliche Erfahrungen gemacht haben und weniger, wenn sie unerfreuliche Erfahrungen gemacht haben (Appiah 2008). Wir mögen dies als moralische Schwäche kritisieren, aber wir müssen solche Zusammenhänge verstehen und können sie nicht einfach ignorieren. Wenn wir z. B. eine Konferenz organisieren, wissen wir, dass die Wahrscheinlichkeit gedeihlicher Interaktionen wächst, wenn wir Kaffee anbieten oder soziale Begegnungsräume eröffnen. Dafür zu sorgen, dass sich andere wohl und wertgeschätzt fühlen, ist eine möglicherweise profane gleichwohl aber unterschätzte Tugend. Auch wenn Rationalist*innen argumentieren mögen, dass es darauf nicht ankommen sollte, legt eine naturalistische Akzeptanz unserer zerbrechlichen sozialen Natur nahe, dass es klüger ist, darauf zu achten. Statt solche gefühlsbezogenen „Schwächen“ als etwas zu verstehen, das wir als rationale Wesen überwinden sollten und dass wir aus dem ,härteren Holz geschnitzt“ sein sollten, etwa im Sinne eines maskulinistischen, bourgeoisen Verständnisses von „Charakter“, sollten wir achtsam gegenüber diesen „Schwächen“ sein und möglichst mit ihnen statt gegen sie arbeiten.

So sehr wir auch möchten, dass unser Verhalten der Vernunft folgt, ist unsere praktische Vernunftfähigkeit durch solche Interaktionen von Denken und Körper beeinflusst. Unsicherheitsgefühle sind nicht förderlich für unsere Fähigkeit, anderen zuzuhören und gute Entscheidungen zu treffen, während ein Gefühl von Sicherheit und Zuversicht das Zuhören, Nachdenken und Problemlösen erleichtert (Porges 2011). Insbesondere, wenn wir andere bewerten, sollten wir all diese sozialen und vorbewussten Einflüsse auf Menschen anerkennen, statt zu versuchen, sie in einem Gehäuse der Vernunft einzuhegen und dabei unsere eigene relativ privilegierte Perspektive ebenso zu ignorieren wie die Tatsache, dass wir selbst ebenfalls nicht frei von diesen Einflüssen - im Guten wie im Schlechten - sind. Wir bedürfen einer psychologischen ebenso wie einer soziologischen Vorstellungskraft. 


\section{Ein Beispiel}

Bevor ich Abschluss komme, möchte ich einige der Fäden dieses Beitrags durch ein Beispiel zusammenbringen. Nick Duffel ist ein Psychotherapeut, der auf die Pathologien parentaler Deprivation in Folge von Internatserziehung ${ }^{3}$ spezialisiert ist. Duffel war selbst Internatsschüler und sein Buch Wounded Leaders (Duffel 2014) beschäftigt sich mit den Folgen dieser Erfahrungen.

Von den alltäglichen Erinnerungen an und der Sicherheit von unkonditionaler elterlicher Liebe abgeschnitten, tendieren Internatsschüler*innen dazu, selbst-verteidigende Persönlichkeiten zu entwickeln, die Verletzlichkeit und Zweifel verdecken, um Schikanen und Bloßstellungen zu vermeiden. Sie tendieren zur Entwicklung einer „strategic survival personality“ für die Instrumentalismus und das Verfolgen von Eigeninteressen die Norm ist, während Schwächen verheimlicht oder geleugnet werden müssen. Sie fürchten und verdrängen ihre eigenen Verwundbarkeiten und tendieren dazu, sie auf andere zu projizieren teilweise auch um sie dort aufzuspüren und eigene Vorteile daraus zu ziehen. Zugleich kommen diese Schüler*innen in den Genuss außergewöhnlicher Einrichtungen und deren inhärenten kulturellen Kapitals. Vor diesem Hintergrund entwickeln sie einen Habitus, der durch einen Berechtigungs- und Überlegenheitssinn charakterisiert ist. Jenen, die es schaffen, diese Erfahrungen zu meistern, erleichtern sie Erfolg in der Karrierewelt der Politik durch die instrumentelle Behandlung anderer, die Unwilligkeit Schwächen zu gestehen oder Zweifel zuzulassen sowie die Tendenz, Angriff als die beste Verteidigung einzusetzen. Diese Form des Aufwachsens tendiert emotionale Illiteralität, die Vermeidung von Intimität und eine Reihe weiter Pathologien hervorzubringen, wie etwa „Splitting“, d.h. die Unterdrückung von Gefühlen, die sie nicht zulassen können und deren Projektion auf andere. Duffel veranschaulicht dies an vielen Beispielen von Verhaltensmerkmalen von Politikern im Vereinigten Königreich.

Das Beispiel der „boarding schools“ bringt viel von dem zusammen, was ich versucht habe, aufzuzeigen: Die Bedeutung von Bindung und Care sowie den $\mathrm{Zu}$ sammenhang von ungleichen gesellschaftlichen Positionen mit der Entwicklung von Habitus, Tugend, Laster und Persönlichkeit. Das Beispiel erschließt sich über ein Verständnis der formativen Einflüsse, sowohl der interpersonalen Beziehungen in Familie und Schule, als auch der Positionen der Individuen im gesamten ungleichen Feld des Sozialen.

\section{Schluss}

Der Beitrag sollte den Wert einer Verbindung von Tugendethik und sozialwissenschaftlicher Forschung herausarbeiten. Der Tugendethik gelingt es besser als den meisten anderen Moralphilosophien, die Bedeutung von Biographie und Erfahrung für die Praktiken von Individuen anzuerkennen. Gleichwohl sollte sie diese Einflüsse systematischer, auf dem Fundament sozialwissenschaftlicher Forschungen zu Ungleichheit und Bindungen untersuchen. Mit Blick auf Ungleichheit ermöglicht

\footnotetext{
3 Gemeint sind hier vor allem die elitären britischen Boarding Schools (Anm. d. Übers.).
} 
uns das Habituskonzept, die Tugenden und Laster von Individuen im Zusammenhang mit ihren Positionen in durch Ungleichheit geprägten Gesellschaften zu verstehen. Mit Blick auf Bindungen verdeutlicht die psychologische Forschung über die Formierung von Dispositionen im Kindesalter die Relevanz der Qualität der Beziehungen zu primären Care-Givern. Es sollte deutlich geworden sein, dass wir, um Tugendenden und Übel im Alltagsleben zu verstehen, eine psycho-soziologische Vorstellungskraft benötigen, die sich dieser Einflüsse auf uns und andere bewusst ist.

Open Access Dieser Artikel wird unter der Creative Commons Namensnennung 4.0 International Lizenz (http://creativecommons.org/licenses/by/4.0/deed.de) veröffentlicht, welche die Nutzung, Vervielfältigung, Bearbeitung, Verbreitung und Wiedergabe in jeglichem Medium und Format erlaubt, sofern Sie den/die ursprünglichen Autor(en) und die Quelle ordnungsgemäß nennen, einen Link zur Creative Commons Lizenz beifügen und angeben, ob Änderungen vorgenommen wurden.

\section{Literatur}

Appiah, K. A. (2008). Experiments in ethics. Cambridge: Harvard University Press.

Benjamin, J. (1988). Bonds of love. New York: Pantheon.

Benner, P. (1994). The role of articulation in clinical nursing. In J. Tully (Hrsg.), Philosophy in an age of pluralism: the philosophy of Charles Taylor in question. Cambridge: Cambridge University Press.

Bourdieu, P. (1986). Distinction: a social critique of the judgement of taste. London: Routledge.

Bourdieu, P. (2000). Pascalian meditations. Cambridge: Polity.

Bowlby, J. (1969). Attachment and loss. Bd. 1: Attachment. New York: Basic Books.

Brennan, G., \& Pettit, P. (2004). The economy of esteem. Oxford: Oxford University Press.

Cassidy, J., \& Shriver, P. R. (1999). Handbook of attachment. New York: Guilford.

Duffell, N. (2014). Wounded leaders: British elitism and the entitlement illusion. London: Lone Arrow.

Dunne, J. (1993). Back to the rough ground: practical judgment and the lure of technique. Indiana: University of Notre Dame Press.

Glover, J. (2001). Humanity: a moral history of the twentieth century. London: Pimlico.

Goodin, R.E. (1985). Protecting the vulnerable: a reanalysis of our social responsibilities. Chicago: University of Chicago Press.

Griswold, C. L. Jr. (1999). Adam Smith and the virtues of enlightenment. Cambridge: Cambridge University Press.

Held, V. (2006). The ethics of care. New York: Oxford University Press.

Kittay, E. F. (1999). Love's labor: essays on women, equality and dependency. New York: Routledge.

Lareau, A. (2003). Unequal childhoods: class, race and family life. Berkeley: University of California Press.

Lynch, K., Baker, J., \& Lyons, M. (2009). Affective equality: Love, care and injustice. New York: Palgrave Macmillan.

MacIntyre, A. (1981). After virtue: a study in moral theory. London: Duckworth.

Murdoch, I. (1970). The sovereignty of good. London: Routledge.

Nussbaum, M.C. (1986). The fragility of goodness. Cambridge: Cambridge University Press.

Nussbaum, M.C. (2001). Upheavals of thought: the intelligence of emotions. Cambridge: Cambridge University Press.

Pink, D. H. (2011). Drive: the surprising truth of what motivates us. New York: Riverhead books.

Porges, S. (2011). The Polyvagal theory: neurophysiological foundations of emotions, attachment, communication and self-regulation. New York: W. W. Norton.

Reay, D. (1998). Class work: mothers' involvement in their children's primary schooling. London: University College London.

Reay, D. (2002). Shaun's story. Gender and Education, 14(2), 221-234.

Sayer, A. (2005). The moral significance of class. Cambridge: Cambridge University Press.

Sayer, A. (2011). Why things matter to people: social science, values and ethical life. Cambridge: Cambridge University Press.

Sevenhuijsen, S. (1998). Citizenship and the ethic of care. London: Routledge.

Siegel, D. J. (1999). The developing mind. New York: Guilford. 
Smith, T. (1984). The theory of moral sentiments. Indianapolis: Liberty Fund.

Tessman, L. (2005). Burdened virtues. Oxford: Oxford University Press.

Tronto, J.C. (1994). Moral boundaries. London: Routledge.

Vetlesen, A. J. (2005). Evil and human agency: understanding collective evildoing. Cambridge: Cambridge University Press.

Winnicott, D. W. (1964). The child, the family, and the outside world. Harmondsworth: Penguin. 